\title{
Research Article \\ Partner Phubbing as a Social Allergen: Support for a Dual Process Model
}

\author{
James A. Roberts $(\mathbb{D}$ ) and Meredith E. David \\ Hankamer School of Business, Baylor University, One Bear Place \#98007, Waco, Texas 76798, USA \\ Correspondence should be addressed to James A. Roberts; jim_roberts@baylor.edu
}

Received 10 January 2022; Accepted 12 February 2022; Published 3 March 2022

Academic Editor: Zheng Yan

Copyright (c) 2022 James A. Roberts and Meredith E. David. This is an open access article distributed under the Creative Commons Attribution License, which permits unrestricted use, distribution, and reproduction in any medium, provided the original work is properly cited.

\begin{abstract}
In a couple context, a social allergen is a behavior that irritates one's partner and tends to increase as a romantic relationship continues. Given that smartphones are a constant companion for many people, their use in the presence of one's romantic partner is pervasive and can have important implications for relationships. The present research focuses on relationship length and partner phubbing and investigates the mediating role of passion and deromantization on the social allergen of partner phubbing. Study 1 surveyed 250 married adults and found that relationship length is negatively associated with romantic passion which, in turn, is positively associated with perceptions of partner phubbing. Although the literature on social allergens would suggest a positive effect of relationship length on partner phubbing, Study 1 showed no significant main effect of relationship length on phubbing. As an attempt to explain this seeming anomaly, we drew from the attachment theory to propose an additional mechanism underlying this relationship. Study $2(n=250$ married adults) then tests an expanded model that includes attachment anxiety as an additional mediator. Results show that relationship length is associated with lower attachment anxiety which is in turn associated with less perceived partner phubbing. Overall, the results show support for a dual process model, such that romantic passion and attachment anxiety differentially underlie the path between relationship length and perceived partner phubbing. The findings provide important insights into better understanding partner phubbing as a social allergen over the course of marital relationships.
\end{abstract}

\section{Introduction}

Navigating romantic relationships is a challenging task. And research suggests that this task becomes increasingly difficult as relationships progress [1]. Social allergens, or more simply "bad habits," tend to increase as a romantic relationship continues [2]. Those initially minor irritants (e.g., talking while your spouse tries to read or watch TV, uncouth habits such as burping or flatulence, or, being ignored while your spouse is captivated by their smartphone) can eventually become major roadblocks to a healthy and happy relationship through the process of repetition sensitization $[3,4]$. Initially, one's spouse being preoccupied with his or her smartphone may only be a mild irritant. But, as this behavior continues, it could elicit a much stronger negative reaction and may undermine a romantic partner's satisfaction with the relationship and even lead to dissolution of the marriage or relationship $[5,6]$.

A social allergen is defined as an "emotion-arousing behavior or situation created by another person that is seen as unpleasant, but not as unbearably aversive, by objective observers" ([2], p. 274). Over time, repeated exposure to such behavior can produce a social allergy. A social allergy is "a reaction of hypersensitive annoyance or disgust to a social allergen" ([2], p. 274).

Partner phubbing (phone snubbing), which is defined as the perceived "extent to which your romantic partner uses or is distracted by his/her cell phone while in your company" ([7], p. 137) may be a social allergen which leads to increasingly negative reactions on the part of the affected romantic partner. Given that smartphones are a constant companion for many people [8], their use in the presence of one's 
romantic partner is pervasive [9] and can have important implications for the relationship satisfaction of the affected partner $[7,10,11]$. Particularly troublesome is the fact that social allergens like partner phubbing are rarely discussed among relationship partners [12]. In their pioneering research on social allergens, Cunningham et al. [2] conclude that social allergens can have a negative effect on relationship satisfaction and even lead to the termination of romantic relationships.

The primary objective of the present research is to investigate the association between the length of romantic relationships and perceived partner phubbing, as well as the processes that underlie this relationship. Extant research has shown the negative implications of perceived partner phubbing on an individual's well-being [7, 13-15], and thus it is important to understand how and why romantic relationships and the duration of these relationships are associated with perceived partner phubbing. In the present research, two interrelated processes, reduced passion and deromantization, are examined as mediators underlying the association between the length of romantic relationships and the perceived incidence of a specific social allergen-partner phubbing. A second study considers an additional, third process and investigates the mediating role that attachment anxiety might play in better understanding the length of relationship_-partner phubbing relationship.

\section{Relationship Length and Partner Phubbing}

Roberts and David [8] have identified partner phubbing as a social allergen. Previous research has found that social allergens increase with the length of romantic relationships [2]. In a small sample of undergraduate students $(n=125)$, Felmlee [6] asked respondents to reflect on their current or a past romantic relationship. As part of an overall larger survey, respondents were asked if the qualities they currently least like about their romantic partner were "very different or very similar to" those that initially attracted them. Several respondents explained how they came to dislike certain behaviors of their romantic partner. One common explanation for their disillusionment with their current romantic partner was labelled "time will tell" by Felmlee [6]. Respondents explained how certain bothersome behaviors performed by their romantic partners were not apparent at the beginning of the relationship. With time, however, these bothersome behaviors became more evident. A limitation of this study was that the average relationship length was only 1.5 years.

Burpee and Langer [16] argued that mindlessness, which overlaps conceptually with social allergens that bother one's romantic partner but are repeated anyway, is associated with marital satisfaction. In relationships, the authors contend, mindlessness is positively related to relationship length. Over time, romantic couples fall into highly routinized behavior and may become less aware of how their behavior is impacting their relationship partner. Lenger, Gordon, and Nguyen [17] conclude that "research has yet to determine whether mindfulness decreases with relationship length." Relatedly, Kaighobadi, Shackelford, and Buss [18] studied spouses' mate retention tactics among married couples during the newly wed stage and again four years into the marriage and found that spouses' performance reports of mate retention tactics decrease after being married for three years. Additionally, Wang, Zhao, and Lei [19] studied the adverse effects of partner phubbing among married and unmarried adults and found that partner phubbing has a stronger harmful effect on individuals in married (vs. unmarried) romantic relationships. Given the above, we offer the following hypothesis:

H1: Length of a romantic relationship will be positively associated with perceived partner phubbing.

\section{The Mediating Roles of Reduced Passion and Deromantization}

The argument that social allergens such as partner phubbing will increase as relationships grow longer is supported by two interrelated processes that are present in nearly all romantic relationships: reduced passion and deromantization [2]. Both of these processes have strong face validity and have been empirically supported as well.

Reduced passion is the lessening of romantic passion as a relationship continues. At the onset of romantic relationships, potentially bothersome behaviors may be overlooked or even seen in a favorable light. Idealizing one's romantic partner at the earliest stages of the relationship is common [20]. Romantic passion may blind an individual to the shortcomings of their romantic partner. In essence, individuals see their romantic partner through "rose-colored glasses" ([6], p. 272). As couples spend more time together, the passion begins to fade or level off, and a more realistic picture, "warts and all," begins to emerge. As one's passion for their romantic partner begins to fade, there is less to buffer the irritation associated with allergenic behaviors. [5, 6] on fatal attractions, or qualities that initially attract an individual to their partner but are later viewed as negative qualities, support the notion that an individual's reaction to a certain behavior by their romantic partner can change over time. For example, in the early stages of a relationship, a romantic partner's constant use of their smartphone may be seen as a sign of popularity but later might be interpreted as a lack of interest in the relationship. Given the above, we offer the following hypothesis:

H2:Passion will mediate the association between length of relationship and perceived partner phubbing such that, relationship length is associated with reduced passion which is then associated with lower perceived partner phubbing.

The second process that is hypothesized to act as a mediator between length of relationship and partner phubbing is referred to as "deromantization." As a romantic relationship continues, there will likely be less effort at impression management from one or both partners. Cunningham et al. [2] found that men were more prone to uncouth behaviors (poor grooming, flatulence, etc.) and norm violations (talking too loudly, drinking too much, etc.), while women were more inconsiderate (chronically late, self-occupied, etc.) and intrusive (jealous behavior, criticizing, giving 
commands, etc.). These behaviors/social allergens in relationships were found to increase in frequency over time.

As a romantic partner is less prone to manage the impression made on his or her romantic partner, he/she is more likely to exhibit behavior that is bothersome to their partner. Although the process of deromantization has both theoretical and empirical support, it might be helpful to think about your current behavior in the presence of your romantic partner. What types of behavior or habits do you exhibit today that you would not have considered in the earlier stages of your relationship? Based upon the above, we offer our third hypothesis:

H3:Deromantization will mediate the association between length of relationship and partner phubbing such that relationship length is positively associated with deromantization which is positively associated with perceived partner phubbing.

Our conceptual model is shown in Figure 1. To test the predictions set forth, we conducted a series of studies. First, we conducted a pretest in order to assess the reliability and validity of the measures that would be used in the main study to assess passion and deromantization. This pretest was necessary since established measures of passion and deromantization do not exist in a conceptual form that would fit the current research; previous scales would need to be adapted for the current research. Next, we conducted the main study (Study 1) to test the predictions set forth in our conceptual model. The results from this study generally support our predictions, but evidence from the data suggests that another process (mediator) may be at work [21, 22]. We expand on this possibility and draw from the attachment theory [23] to suggest that interpersonal attachment anxiety could be a key mechanism underlying the link between relationship duration and perceived partner phubbing. We then conducted an additional study (Study 2) to test this prediction, as well as those tested in Study 1, as an attempt to better understand the association between length of romantic relationships and partner phubbing.

3.1. Pretest. A pretest was conducted in the spring of 2021 to assess the reliability of the reduced passion and deromantization measures. The pretest was determined to be EXEMPT from review by the authors' University Institutional Review Board (IRB) according to federal regulation 45 CFR 46.104(d)(2): Research involving the use of educational tests, survey procedures, interview procedures, or observation of public behavior. Across the three studies used in the present research, all study participants completed a consent form before participating in the study. The consent form advised participants of the benefits of participating in the research, the minimal risks associated with their participation, and the anonymity of their responses. Participants were also informed of the voluntary nature of the research, such that participants would not be penalized in any way for choosing to withdraw from or discontinue their participation in the study at any point in time.

Following Cunningham et al. [2], deromantization refers to "a gradual reduction in impression management efforts to be romantically appealing" and is operationalized as an indi- vidual's perception of his/her spouse engaging in bothersome or annoying behaviors more frequently over the course of the relationship. Following this conceptualization, we created nine items to assess deromantization. Example items include, "My spouse engages in less self-monitoring at this point in our relationship than when we first got together," "Over time, my spouse's bad habits get worse," and "My spouse's bothersome habits have worsened over the course of our relationship."

Romantic passion is operationalized as an individual's level of passion towards his/her spouse. Unlike deromantization, which assesses one's perceptions of his/her spouse's behaviors, passion is conceptualized from a self-perspective and assesses one's own feelings as they relate to his/her spouse. This conceptualization is consistent with Cunningham's [2] conceptualization that romantic passion dissipates over time in many relationships, as noted by weaker positive feelings and dissipation of any earlier blissful ignorance. Following this conceptualization, we constructed seven statements aimed at operationalizing romantic passion in a manner consistent with Cunningham's (2005) definition. Example items included, "The love I have for my spouse burns as bright now as when we first got together," "I am not as romantically inclined toward my spouse as when we first got together" (R), and "My relationship is not as passionate as when we first got married" (R).

The pretest sample included 75 US adults (56\% male, $M_{\text {age }}=37$ ) who were currently in a romantic relationship. Participants were recruited and completed the Qualtrics survey using CloudResearch's TurkPrime platform (https://www .cloudresearch.com/). The deromantization and passion scales were both assessed using 5-point Likert scales. Of note, deromantization is assessed as one's perspective of his/her spouse's behaviors, whereas passion is assessed as one's own feelings in the relationship with his/her spouse. The complete list of items used to assess both measures is provided in the Appendix.

A factor analysis was conducted for the 9-item deromantization measure and the 7-item romantic passion measure. The data was well-suited for a factor analysis as indicated by the KMO statistic (.89) and Bartlett's test of sphericity $\left(X^{2}=1085.47, p<.01\right)$. Principal components extraction and varimax rotation were used to interpret the factor loadings [24]. The results reveal the expected two-factor structure, explaining 71 percent of the variance in total. The deromantization measure exhibited a factor structure consistent with the expected one factor conceptualization of the measure, as all nine items loaded onto a single factor. In addition, the results for deromantization revealed a construct reliability estimate of .95 and an average variance extracted of .67, thus showing evidence of the measure's reliability and convergent validity [25]. Similarly, the passion measure exhibited a one-factor structure with all seven items loading onto a single factor; the results for passion revealed a construct reliability estimate of .88 and an average variance extracted of .52, thus showing evidence of the measure's reliability and validity [25]. In addition, and in providing evidence for discriminant validity as recommended by Fornell and Larcker [25], the AVE for each factor exceeded the respective squared correlation between factors. Overall, the 


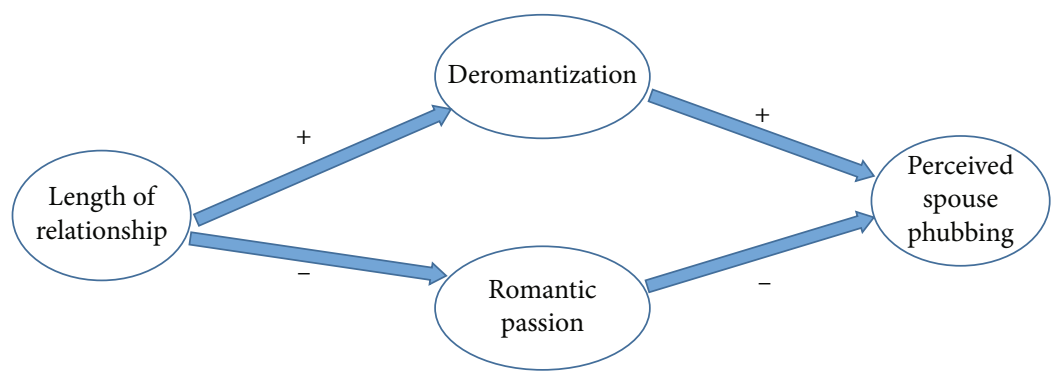

Figure 1: Conceptual model tested in Study 1.

pretest results indicate that the deromantization $(M=2.77$; $\mathrm{SD}=1.13 ; \alpha=.96)$ and passion $(M=3.47 ; \mathrm{SD}=1.01 ; \alpha=$ .90) measures are valid and reliable. Next, we use these measures in Study 1 which is designed to test the conceptual model shown in Figure 1.

3.2. Study 1. Study 1 consisted of 250 adults in the US ( $43 \%$ male, $M_{\text {age }}=43$, Range $=23-82$ ) who were recruited to participate in the study using the Cloud Research.com MTurk Toolkit [26]. The study took place in the late spring of 2021. This study was determined to be EXEMPT from review by the authors' University IRB according to federal regulation 45 CFR 46.104(d)(2): Research involving the use of educational tests, survey procedures, interview procedures or observation of public behavior.

The online survey was constructed in Qualtrics' survey platform and was administered for data collection through CloudResearch which is often referred to by academics as TurkPrime. As noted on their website, "CloudResearch, formerly TurkPrime, is the leading participant-sourcing platform for online research and surveys. Our tools provide academic and market researchers immediate access to millions of diverse, high-quality respondents around the world" (https://www.cloudresearch.com/). CloudResearch (TurkPrime) is a research and survey platform that runs on the Internet through any browser without the need for any software or other downloads; the platform offers high quality crowdsourcing data collection and has been used for many research studies published in highly regarded and top-tier academic journals (e.g., [27]; [28]; [29]; [7, 8, 26, 30].

The data generated from an online survey administered through CloudResearch comes from a large panel of people who have granted prior consent to participate in surveys. The integrity of data from CloudResearch (TurkPrime) has been supported in many previous studies and such samples may well be of higher quality than community samples; evidence has shown that participants from this platform tend to value their ability to contribute to scientific studies on real phenomena, whereas participants from community samples may be more prone to respond in ways that they believe will help the researchers without regard to the integrity of the data $([8,31]$. In addition, research comparing data from the CloudResearch samples, student samples, and community samples has shown that samples from the CloudResearch platform are no more prone to problematic respondent behavior than that from other samples ([32, 33]; [34]; [35]; [31, 36]).
In CloudResearch, researchers are able to specify criteria that must be met for participants to be deemed eligible for participation in the study. We followed standard procedures used in psychological and consumer research to administer questionnaires to participants who met the following criteria: live in the US, at least 18 years of age, and have at least a 95\% approval rating. The sample was also limited to participants who were currently married; 19 participants reported not being married and were thus removed from the sample, resulting in a final sample of 231 married adults.

Participants were asked to think of their spouse as they responded to the main questions in the survey. Romantic passion $(M=3.54 ; \mathrm{SD}=1.04 ; \alpha=.90)$ was measured using the seven-item measure discussed above in the pretest and shown in the Appendix. Similarly, deromantization $(M=2.66 ; \mathrm{SD}=.99 ; \alpha=.95)$ was measured using the nineitem measure discussed above and shown in the Appendix. A 5 -point Likert scale $(1=$ strongly disagree, $5=$ strongly agree) was used as the response format for the passion and deromantization scales.

Partner phubbing $(M=2.86 ; \mathrm{SD}=.92 ; \alpha=.92)$ was assessed using the 9-item measure by Roberts and David [7]. Example items include "During a typical mealtime that my spouse and I spend together, my partner pulls out and checks his/her phone," and "My spouse keeps his/her cellphone where he/she can see it when we are together." A five-point response scale was used for the nine partner phubbing items (ranging from never to all of the time). A higher score means higher perceived phubbing. Demographic variables were assessed at the end of the survey.

\section{Descriptive Statistics, Analyses, and Results}

Table 1 shows the descriptive statistics and correlations between the study variables. In terms of the sample statistics, most participants (43\%) reported having been in their relationship for over 15 years: $24 \%$ for $10-15$ years; $17 \%$ for $6-9$ years; and $16 \%$ for $1-5$ years. Most participants were Caucasian (74\%), followed by Hispanic (11\%), African American (9\%), and then Asian (5\%). Seven percent of participants had a high school diploma, 18\% had some college, 55\% had a college degree, and $20 \%$ had a master's/doctoral degree.

The majority of participants were employed full-time (64\%), with $17 \%$ employed part-time, $10 \%$ being homemakers, $7 \%$ being retired, $2 \%$ not employed, and $1 \%$ of the sample was students. The most frequent annual household income ranges among participants were $\$ 75,000-\$ 99,999$ (26\%), followed by 
TABle 1: Study 1 descriptive statistics and correlations between study variables.

\begin{tabular}{lcccccc}
\hline Variable & $n$ & $M$ & $\mathrm{SD}$ & 1 & 2 & 3 \\
\hline 1. LOR & 231 & 5.94 & 1.16 & & & \\
2. DR & 231 & 2.66 & .99 & $.208^{* *}$ & & \\
3. Passion & 231 & 3.54 & 1.04 & $-.229^{* *}$ & $-.655^{* *}$ & \\
4. Phubbing & 231 & 2.86 & .92 & -.030 & $.272^{* *}$ & $-.286^{* *}$ \\
\hline
\end{tabular}

Note. $\mathrm{LOR}=$ length of relationship; $\mathrm{DR}=$ deromantization; Phubbing $=$ partner phubbing. LOR was assessed on scale from 1 to 7 . Correlation coefficients shown in cells. ${ }^{*} p<.05 ;{ }^{* *} p<.01$.

$\$ 100,000-\$ 150,000(22 \%)$ and $50,000-\$ 74,999(22 \%)$, and then over \$150,000 (9\%), \$40,000-\$49,999 (8\%), \$30,000-\$39,999 (7\%), $\$ 20,000-\$ 29,000(4 \%)$, and less than $\$ 20,000(2 \%)$.

Since the data for all measures were obtained from the same source, common method variance could bias the results [37]. As such, we performed the Lindell and Whitney [38] marker variable procedure. Specifically, two items that were expected to be theoretically unrelated to the measures used in the study (e.g., "how satisfied are you with the weather outside today?") were embedded in the questionnaire. The correlations between the marker variable items and each of the study measures were nonsignificant, small, and close to zero. Therefore, it is unlikely that common method bias affected the results [38, 39].

The Preacher and Hayes [21] PROCESS bootstrapping SPSS macro model 4 was used to test the predictions in our conceptual model. The Preacher and Hayes [21] PROCESS macro for SPSS uses an ordinary-least-squares path analysis to estimate model coefficients and to assess the indirect and/or direct effects of relationship length on perceived partner phubbing $([40,41]$. The PROCESS models use a bootstrapping procedure $(n=5000)$, which does not rely on any assumptions about the normality of the sampling distribution underlying the indirect effect, to calculate the biascorrected $95 \%$ confidence intervals associated with the statistical significance of the indirect effects [21, 42]. Of note, participant's age was included as a covariate in the analyses presented below; analyses without age as a covariate showed results consistent with those presented below.

First, the model tests the link between relationship length and deromantization $\left(F_{2,228}=5.44, p<.01, R^{2}=.05\right)$. The results show that length of relationship is positively associated with deromantization $(\beta=.17, p=.03)$. Next, the model tests whether relationship length is directly associated with romantic passion. The results $\left(F_{2,228}=8.01, p<.001, R^{2}=.07\right)$ indicate that relationship length is negatively associated with romantic passion $(\beta=-.17, p<.05)$, suggesting that passion decreases over time in relationships.

Next, the model tests whether relationship length, deromantization, and romantic passion are significant predictors of perceived partner phubbing $\left(F_{4,226}=8.77, p<.001, R^{2}=\right.$ .13). The results indicate that length of the relationship $(\beta=.01, p=.86)$ is not significantly associated with perceived partner phubbing; deromantization $(\beta=.16, p=.06)$ is marginally associated with perceived partner phubbing.
However, passion $(\beta=-.22, p=.01)$ is negatively associated with perceived partner phubbing, and the results show support for mediation. Specifically, and in support of H2, relationship length has a significant and positive indirect association with perceived partner phubbing through romantic passion $(\beta=.04, p<.05,95 \%$ CI: .001, .091). However, the indirect effect of relationship length on perceived partner phubbing $(\mathrm{H} 3)$ is not significant through deromantization $(\beta=.03, p>.05,95 \%$ CI: $-.004, .077)$.

Overall, the mediation results reveal that relationship length has a positive indirect effect on partner phubbing through romantic passion. That is, longer relationships are associated with lower levels of passion which increases perceived partner phubbing. Deromantization, however, did not have a significant mediating role between relationship length and perceived partner phubbing. Of note, an interesting and unexpected finding from this study relates to the lack of a significant main effect of relationship length on partner phubbing. It was predicted in $\mathrm{H} 1$ that length of the relationship would be positively associated with perceived partner phubbing, but the study results showed no significant main effect. We sought to further examine this relationship in order to gain a better understanding of why the expected positive main effect did not show up in the study results. Drawing from seminal research in methodology and the PROCESS method for testing multiple mediation models $[21,22]$, it seems likely that this unexpected result (or lack thereof) could be due to another process (mediator) being at work. We expand on this possibility by drawing from the attachment theory [23]. Specifically, and as discussed next, it is likely that attachment style could be another key mechanism that mediates the link between relationship duration and perceived partner phubbing.

4.1. The Mediating Role of Attachment Anxiety. Drawing from the attachment theory [23], it is likely that interpersonal attachment style, and attachment anxiety particularly could assist in explaining why a positive main effect of relationship length on perceived partner phubbing was not found in Study 1. The literature on attachment theory explains that individuals develop an attachment style based on the caregiving received in early relationships [23]. An individual's attachment style continues to develop as a person matures into adulthood and eventually engages in romantic relationships [43].

Research has shown that attachment style impacts individuals' perceptions of support from others [23, 44, 45]. Anxiously attached individuals are hypersensitive to their personal interactions and have a heightened desire for support from others as they fear being abandoned or rejected by close others $[43,46])$. Avoidantly attached individuals, however, are self-reliant and do not have a hypersensitivity to receiving support from others. Thus, it is attachment anxiety in particular that is likely associated with perceived phubbing, and more of it, as anxiously attached individuals are particularly concerned with maintaining closeness in relationships [45]. In seeking and attempting to maintain attention from others, anxiously attached individuals engage in hyperactivating strategies; these strategies are characterized 
by heightened sensitivity to and preoccupation with threatrelated actions as well as immediate detection of relational threats and a constant clinging to others [47]. Thus, it is likely that attachment anxiety is positively associated with perceived partner phubbing.

In addition, relationship length should be negatively associated with attachment anxiety, such that attachment anxiety declines over the course of a romantic relationship. Indeed, attachment theorists have shown that, although parental attachment styles are relatively stable over the course of childhood, romantic attachment styles, or attachment to one's romantic partner, are less stable, with individuals in committed romantic relationships or marriages generally becoming more secure and less anxiously attached over time [48-51]. Based on the literature discussed above, we predict the following:

H4:Attachment anxiety will mediate the relationship between length of relationship and partner phubbing such that, as a relationship grows longer, attachment anxiety will lessen, and perceived partner phubbing will decline.

Next, we conducted an additional study to examine the role of attachment anxiety as an additional mediator between relationship length and perceived partner phubbing as shown in Figure 2. Specifically, Study 2 tests the same predictions as Study 1 while also testing the potential mediating role of attachment anxiety. It is likely, based on the above review, that a dual process model underlies the link between relationship length and perceived partner phubbing.

4.2. Study 2. Study 2 consisted of 250 adults in the US ( $44 \%$ male, $M_{\text {age }}=43$, Range $\left.=20-76\right)$ who were recruited to participate in the study through the CloudResearch.com MTurk Toolkit [26] using the same qualification criteria as in Study 1. The study took place in the summer of 2021. As with Study 1, this study was also determined to be EXEMPT from review by the authors' University IRB according to federal regulation 45 CFR 46.104(d)(2). The sample was limited to participants who were currently married; 22 participants reported not being married and were thus removed from the sample, resulting in a final sample of 228 married adults. Participants were asked to think of their current romantic partner as they responded to the main questions in the survey. The average length of relationship was 17.51 years (Range $=1-57, \mathrm{SD}=11.63$ ).

The same measures as in Study 1 were used in the present study to assess deromantization $(M=2.48 ; \mathrm{SD}=1.08 ; \alpha=.95)$, romantic passion $(M=3.56 ; \mathrm{SD}=1.01 ; \alpha=.91)$, and perceived spouse phubbing $(M=3.00 ; \mathrm{SD}=.97 ; \alpha=.93)$. Attachment style was assessed using the Experiences in Close Relationships 12-item version (ECR-12) by Lafontaine and colleagues [52] in which six items assess attachment avoidance $(M=2.13$; $\mathrm{SD}=1.26 ; \alpha=.93)$, and six items assess attachment anxiety $(M=2.74 ; S D=1.56 ; \alpha=.92)$ (a 7-point Likert scale was used as the response format). Example items to assess attachment anxiety include, "I worry a fair amount about losing my partner," and "If I cannot get my partner to show interest in me, I get upset or angry." The ECR-12 has been shown to have superior psychometric properties and predictive validity as compared to the leading other measures of attachment style, particularly when assessing attachment to one's partner in a romantic relationship (see [27] for a full review of attachment style measures). It has also been used in recent attachment style research [53-55]. At the end of the survey, demographic variables were assessed.

4.3. Descriptive Statistics, Analyses, and Results. Table 2 shows the descriptive statistics and correlations between the study variables. In terms of the sample, most participants were Caucasian (78\%), followed by Asian (9\%), African American (8\%), Hispanic (4\%), and then Native American (1\%). Ten percent of participants had a high school diploma, $18 \%$ had some college, $46 \%$ had a college degree, and $26 \%$ had a master's/doctoral degree. The majority of participants were employed full-time (67\%), with $13 \%$ employed parttime, $9 \%$ being homemakers, $7 \%$ being retired, and $4 \%$ were not employed. The most frequent annual household income ranges among participants were 50,000-\$74,999 (23\%), $\$ 75,000-\$ 99,999(22 \%)$, and $\$ 100,000-\$ 150,000 \quad(22 \%)$, followed by over $\$ 150,000(12 \%), \$ 40,000-\$ 49,999(8 \%)$, $\$ 30,000-\$ 39,999(6 \%), \$ 20,000-\$ 29,000(4 \%)$, and less than $\$ 20,000(3 \%)$.

In order to assess common method bias, we included two theoretically unrelated questions in the survey. As with Study 1 , the findings show that the correlations between the marker variable items and each of the study measures were nonsignificant, small, and close to zero; thus, it is unlikely that common method bias affected the results [38, 39].

The Preacher and Hayes [21] PROCESS bootstrapping SPSS macro model 4 was used to test the predictions discussed above. As in Study 1, age was included as a covariate in the analyses presented below. First, the model tests the impact of relationship length on deromantization $\left(F_{2,225)}=1.35, p>.05\right.$, $\left.R^{2}=.01\right)$. The results show that length of relationship is not significantly associated with deromantization $(\beta=.13, p=.15)$. Next, the model tests whether relationship length is directly associated with passion. The results $\left(F_{2,225}=5.64, p<.01\right.$, $\left.R^{2}=.05\right)$ indicate that relationship length is negatively associated with romantic passion $(\beta=-.26, p=.01)$. Next, the model tests whether relationship length is directly associated with attachment anxiety. The results $\left(F_{2,225}=7.94, p<.01\right.$, $\left.R^{2}=.07\right)$ indicate that relationship length is negatively associated with attachment anxiety $(\beta=-.22, p=.02)$, suggesting that longer relationships are associated with less attachment anxiety.

Next, the model tests whether relationship length, deromantization, passion, and attachment anxiety are significant predictors of perceived partner phubbing. The results $\left(F_{5,222}=16.12, p<.01, R^{2}=.27\right)$ indicate that length of relationship is not directly associated with partner phubbing $(\beta=.07, p=.41)$, but deromantization $(\beta=.17, p=.03)$, passion $(\beta=-.25, p<.01)$, and attachment anxiety $(\beta=.15$, $p=.01)$ are all significantly associated with perceived partner phubbing. Importantly, the results show support for mediation through romantic passion and attachment anxiety. Specifically, and consistent with the findings from Study 1, relationship length has a positive indirect effect on perceived phubbing through romantic passion $(\beta=.07, p<.05,95 \%$ 


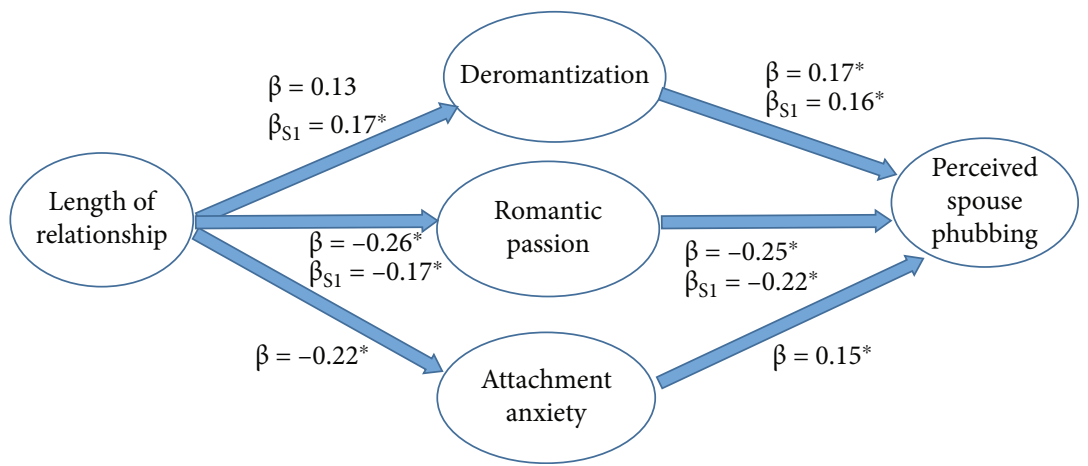

FIgURe 2: Study 1 and 2 results.

TABLE 2: Study 2 descriptive statistics and correlations between study variables.

\begin{tabular}{|c|c|c|c|c|c|c|c|}
\hline Variable & $n$ & $M$ & SD & 1 & 2 & 3 & 4 \\
\hline 1. Anxiety & 228 & 2.74 & 1.56 & & & & \\
\hline 2. LOR & 228 & 17.51 & 11.63 & $-.235^{* *}$ & & & \\
\hline 3. DR & 228 & 2.48 & 1.08 & $.276^{* *}$ & .019 & & \\
\hline 4. Passion & 228 & 3.56 & 1.01 & -.075 & $-.189^{* *}$ & $-.634^{* *}$ & \\
\hline 5. Phubbing & 228 & 3.00 & .97 & $.263^{* *}$ & $-.134^{*}$ & $.395^{* *}$ & $-.355^{* *}$ \\
\hline
\end{tabular}

Note. $\mathrm{LOR}=$ length of relationship; $\mathrm{DR}=$ deromantization; Phubbing = partner phubbing; LOR was measured using a ratio scale. Correlation coefficients shown in cells. $* p<.05 ; * * p<.01$.

CI: .012, .134). In addition, the results show that relationship length has a negative indirect effect on spouse phubbing through attachment anxiety $(\beta=-.03, p<.05,95 \% \mathrm{CI}$ : $-.086,-.001)$. The indirect effect of relationship length on perceived partner phubbing is not significant through deromantization $(\beta=.02, p>.05,95 \% \mathrm{CI}:-.010, .078)$, consistent with Study 1 .

Overall, the results from Study 2 are consistent with the findings from Study 1 and also add to the results of Study 1 by revealing a dual process model which demonstrates how relationship length can have a positive or negative effect on perceived partner phubbing. Importantly, the results support for two different forms of mediation. Firstly, and consistent with the Study 1 findings, the results show that passion decreases over time in relationships and this lower passion is associated with higher perceived partner phubbing. Secondly, the results indicate that longer relationships are associated with less attachment anxiety, and lower attachment anxiety is associated with lower perceived partner phubbing. In sum, Study 2 provides additional support for the results found in Study 1 and adds to the findings from Study 1 by showing that attachment anxiety is a key mediator between relationship length and perceived partner phubbing.

\section{Discussion}

We all have certain habits that irritate our romantic partners. These social allergens can become increasingly problematic as relationships continue. Such social allergens can vary widely from uncouth behaviors, norm violations, intru- sive, and inconsiderate behaviors ([2, 8]. Given the deeply embedded nature of smartphones in every aspect of modern life and the importance of healthy romantic relationships, the present studies investigated the association between length of marital relationships and perceived partner phubbing. This research is the first to investigate the role of romantic passion and deromantization on the social allergen of partner phubbing among married couples.

Research suggests that a general decrease in impression management efforts (deromantization) and a reduction in romantic passion are nearly inevitable consequences of lengthening marital relationships [2]. One important contribution of the present research is the creation of two scales to measure romantic passion and deromantization, respectively. Both scales were found to be valid and highly reliable across studies (alphas $=.90$ to .95). These scales will be helpful in future research efforts that focus on the impact of length of relationships on the development of social allergens. Partner phubbing, as a social allergen, is an important area of research given previous research has shown that partner phubbing can undermine relationship satisfaction and individual well-being as well as lead to relationship termination (e.g., [7, 9, 56, 57].

Study 1 found that romantic passion mediates the link between length of relationship and perceived partner phubbing. As marital relationships progress, romantic passion decreases which, in turn, is associated with increased perceived partner phubbing. Deromantization was not found to mediate the length of relationship-partner phubbing relationship. Cunningham et al. [2] labeled deromantization and reduced passion as interrelated processes. It appears that the 
reduction in romantic passion associated with lengthening marital relationships is an important conduit which drives perceptions of partner phubbing. It may be that a loss of passion that is associated with most romantic relationships also reflects a lessening concern with impression management.

Drawing from Cunningham's [2] theorizing on social allergens, we hypothesized that, as romantic relationships progressed, so too would partner phubbing as a social allergen. The underlying processes according to this theoretical framework were predicted to be deromantization and reduced romantic passion. However, the results showed support for just one of these processes-romantic passion. In effort to gain a better understanding of the processes underlying the link between relationship length and perceived partner phubbing, we conducted a second study which drew from an alternate stream of research in the romantic relationships literature which suggests that beneficial outcomes arise from romantic relationships as they progress. Specifically, we drew from the attachment theory [23] to explain how attachment anxiety could play a mediating role between length of relationship and perceived partner phubbing. Study 2 tested this proposed dual process model such that relationship length was posited to have a positive indirect effect on perceived partner phubbing through reduced romantic passion but a negative indirect effect on perceived phubbing through reduced attachment anxiety. Results suggest that marital relationship duration is associated with less attachment anxiety, and since attachment anxiety is positively associated with perceived partner phubbing, lower attachment anxiety in marital relationships was found to be associated with less perceived phubbing. It appears that higher attachment anxiety is associated with a greater sensitivity to a spouse's phone use as a possible sign of relational devaluation.

\section{Future Research}

Given the complexity and natural evolution of marital relationships, other aspects of a marital relationship must be added to the current model. Cunningham et al. [2] suggest that including positive partner behaviors in one's model may better reflect the ultimate outcomes of social allergens on marital relationships. Kindness and thoughtfulness directed at one's romantic partner may counterbalance the array of "bad habits" many spouses exhibit. Relatedly, future research should examine potential moderators of the mediating paths examined in the present research to determine when one process is likely to be more operative than the other.

The paucity of research on the evolution of social allergens over the course of romantic relationships also needs to be addressed. Although the present research was the first to investigate the association between length of relationship and the social allergen of partner phubbing through the possible mediators of romantic passion, deromantization, and attachment anxiety, a limitation of the research is that both studies were correlational in nature. Given the introduction of two new measures and the first investigation of partner phubbing as a social allergen, the lack of a causal research design is understandable. Future research, however, will benefit from longitudinal and/or experimental research designs that study the development of and/or changing role social allergens might play as marital relationships continue. It is likely that these irksome behaviors may become major annoyances as relationships progress.

Future research is also needed that investigates the role of gender in the relationship between partner phubbing and relationship satisfaction. To date, the empirical results have been somewhat mixed. Khodabakhsh and Ong [58] found that females reported lower levels of marital quality than their male counterparts when phubbed by their romantic partner. Chotpitayasunondh and Douglas [59] found that gender moderated the relationship between being phubbed and viewing phubbing as a normal occurrence. In a sample of Ukrainian college students, Ivanova et al. [60] found that the impact of loneliness on the mediating role of phubbing in the relationship between mobile phone addiction and depression was moderated by gender. Aljasir [10], however, found no moderating role for gender in the relationship between partner phubbing and relationship satisfaction. Given the important role of gender across a wide variety of behaviors, it is imperative that future research investigates how it impacts the outcomes of partner phubbing in romantic relationships.

Research by Birditt, Fingerman, and Almeida [61] found that overall older adults report fewer interpersonal tensions. However, when older adults (compared to younger adults) do report interpersonal tensions, they are more likely to occur with spouses. The researchers also found that older adults reported that these interpersonal tensions were less problematic than their younger counterparts. Younger adults were more likely to cope with such tensions in a less productive manner. Kohdabakhsh and Ong [58] found that younger married adults reported a stronger negative association between partner phubbing and marital quality than older married adults. These findings appear especially problematic for younger adults in relationships given their heavy smartphone use. Future research is needed that investigates the ways in which romantic partners cope with interpersonal tensions like partner phubbing over the course of their relationships.

\section{Appendix}

\section{Romantic Passion Scale Items}

(1) The love I have for my spouse burns as bright now as when we first got together

(2) I am not as romantically inclined toward my spouse as when we first got together. (R)

(3) My relationship is not as passionate as when we first got married. (R)

(4) I like to be as physically close to my spouse now as I did earlier in our relationship

(5) The emotional connection I once felt with my spouse has diminished over time. (R) 
(6) I feel less passion towards my spouse now days than when we first got married. (R)

(7) I find my spouse to be as sexually attractive now as I did early in our romantic relationship

\section{Deromantization Scale Items}

(1) My spouse engages in certain bothersome habits that he/she did not engage in earlier in our relationship

(2) My spouse does not mind his/her manners like he/ she once did early on in our relationship

(3) My spouse engages in less self-monitoring at this point in our relationship than when we first got together

(4) Over time, my spouse's bad habits get worse

(5) My spouse does more things that bother me now than when we first started dating

(6) My spouse's bothersome habits have worsened over the course of our relationship

(7) My spouse no longer monitors his/her habits like when we first got together

(8) My spouse has more annoying habits today than when we first got married

(9) My spouse has had a gradual decrease in impression management efforts to be romantically appealing

\section{Data Availability}

The data is available from the authors upon request.

\section{Conflicts of Interest}

The authors declare that they have no conflicts of interest.

\section{References}

[1] T. L. Huston, J. P. Caughlin, R. M. Houts, S. E. Smith, and L. J. George, "The connubial crucible: newlywed years as predictors of marital delight, distress, and divorce," Journal of Personality and Social Psychology, vol. 80, no. 2, pp. 237-252, 2001.

[2] M. R. Cunningham, S. R. Shamblen, A. P. Barbee, and L. K. Ault, "Social allergies in romantic relationships: behavioral repetition, emotional sensitization, and dissatisfaction in dating couples," Personal Relationships, vol. 12, no. 2, pp. 273295, 2005.

[3] P. H. Blaney, "Affect and memory: a review," Psychology Bulletin, vol. 99, no. 2, pp. 229-246, 1986.

[4] M. R. Cunningham, A. P. Barbee, and P. B. Druen, "Social alleregns and the reactions that they produce: Escalation of annoyance and disgust in love and work," in Aversive Interpersonal Behaviors, R. M. Kowalski, Ed., pp. 190-215, Plenum, New York, 1997.

[5] D. H. Felmlee, "Fatal attractions: affection and disaffection in intimate relationships," Journal of Social and Personal Relationships, vol. 12, no. 2, pp. 295-311, 1995.
[6] D. H. Felmlee, "From appealing to appalling: disenchantment with a romantic partner," Sociological Perspectives, vol. 44, no. 3, pp. 263-280, 2001.

[7] J. A. Roberts and M. E. David, "My life has become a major distraction from my cell phone: partner phubbing and relationship satisfaction among romantic partners," Computers in Human Behavior, vol. 54, pp. 134-141, 2016.

[8] J. A. Roberts and M. E. David, "Partner phubbing and relationship satisfaction through the lens of social allergy theory," Personality and Individual Differences, 2022, Under review.

[9] V. Chotpitayasunondh and K. M. Douglas, "The effects of "phubbing" on social interaction," Journal of Applied Social Psychology, vol. 48, no. 6, pp. 304-316, 2018.

[10] S. Aljasir, "Present but absent in the digital age: testing a conceptual model of phubbing and relationship satisfaction among married couples," Human Behavior and Emerging Technologies, vol. 2022, pp. 1-11, 2022.

[11] B. T. McDaniel, A. M. Galovan, and M. Drouin, "Daily technoference, technology use during couple leisure time, and relationship quality," Media Psychology, vol. 24, no. 5, pp. 637-665, 2021.

[12] B. P. O'Connor, "16 Social Allergens," in Handbook of interpersonal psychology: Theory, research, assessment, and therapeutic interventions, pp. 269-280, Wiley, 2011.

[13] Y. Al-Saggaf and S. B. O’Donnell, "Phubbing: perceptions, reasons behind, predictors, and impacts," Human Behavior \& Emerging Technology, vol. 1, no. 2, pp. 132-140, 2019.

[14] M. E. David and J. A. Roberts, "Investigating the impact of partner phubbing on romantic jealousy and relationship satisfaction: the moderating role of attachment anxiety," Journal of Social and Personal Relationships, vol. 38, no. 12, pp. 35903609, 2021.

[15] X. Wang, X. Xie, Y. Wang, P. Wang, and L. Lei, "Partner phubbing and depression among married Chinese adults: the roles of relationship satisfaction and relationship length," Personality and Individual Differences, vol. 110, pp. 12-17, 2017.

[16] L. C. Burpee and E. J. Langer, "Mindfulness and marital satisfaction," Journal of Adult Development, vol. 12, no. 1, pp. 43-51, 2005.

[17] K. A. Lenger, C. L. Gordon, and S. P. Nguyen, "A word to the wise: age matters when considering mindfulness in romantic relationships," Contemporary Family Therapy, vol. 41, no. 2, pp. 115-124, 2019.

[18] F. Kaighobadi, T. K. Shackelford, and D. M. Buss, "Spousal mate retention in the newlywed year and three years later," Personality and Individual Differences, vol. 48, no. 4, pp. 414-418, 2010.

[19] X. Wang, F. Zhao, and L. Lei, "Partner phubbing and relationship satisfaction: self-esteem and marital status as moderators," Current Psychology, vol. 40, no. 7, pp. 3365-3375, 2019.

[20] S. Niehus, A. Reifman, and C. R. Oldham, "Effects of relational transgressions on idealization of and disillusionment with one's romantic partner: a three-wave longitudinal study," Personal Relationships, vol. 26, no. 3, pp. 466-489, 2019.

[21] K. J. Preacher and A. F. Hayes, "Asymptotic and resampling strategies for assessing and comparing indirect effects in multiple mediator models," Behavior Research Methods, vol. 40, no. 3, pp. 879-891, 2008.

[22] X. Zhao, J. G. Lynch, and Q. Chen, "Reconsidering Baron and Kenny: myths and truths about mediation analysis," Journal of Consumer Research, vol. 37, no. 2, pp. 197-206, 2010. 
[23] J. Bowlby, Attachment and Loss: Vol. 1 Attachment, Basic Books, New York, NY, 2nd edition edition, 1969.

[24] K. L. Haws, U. M. Dholakia, and W. O. Bearden, "An assessment of chronic regulatory focus measures," Journal of Marketing Research, vol. 47, no. 5, pp. 967-982, 2010.

[25] C. Fornell and D. F. Larcker, "Evaluating structural equation models with unobservable variables and measurement error," Journal of Marketing Research, vol. 18, no. 1, pp. 39-50, 1981.

[26] L. Litman, J. Robinson, and T. Abberbock, "TurkPrime.com: a versatile crowdsourcing data acquisition platform for the behavioral sciences," Behavior Research Methods, vol. 49, no. 2, pp. 433-442, 2017.

[27] M. E. David, K. Carter, and C. Alvarez, "An assessment of attachment style measures in marketing," European Journal of Marketing, vol. 54, no. 12, pp. 3015-3049, 2020.

[28] M. E. David, W. O. Bearden, and K. L. Haws, "Priced just for me: The role of interpersonal attachment style on consumer responses to customized pricing," Journal of Consumer Behaviour, vol. 16, no. 6, pp. e26-e37, 2017.

[29] S. Davidai, "Why do Americans believe in economic mobility? Economic inequality, external attributions of wealth and poverty, and the belief in economic mobility," Journal of Experimental Social Psychology, vol. 79, pp. 138-148, 2018.

[30] K. Mortensen and T. L. Hughes, "Comparing Amazon's mechanical Turk platform to conventional data collection methods in the health and medical research literature," Journal of General Internal Medicine, vol. 33, no. 4, pp. 533-538, 2018.

[31] E. A. Necka, S. Cacioppo, G. J. Norman, and J. T. Cacioppo, "Measuring the prevalence of problematic respondent behaviors among MTurk, campus, and community participants," PLoS One, vol. 11, no. 6, article e0157732, 2016.

[32] J. Chandler, P. Mueller, and G. Paolacci, "Nonnaïveté among Amazon mechanical Turk workers: consequences and solutions for behavioral researchers," Behavior Research Methods, vol. 46, no. 1, pp. 112-130, 2014.

[33] M. Crump, J. C. John, V. McDonnell, and T. M. Gureckis, "Evaluating Amazon's mechanical Turk as a tool for experimental behavioral research," PLoS One, vol. 8, no. 3, article e57410, 2013.

[34] J. J. Horton, D. G. Rand, and R. J. Zeckhauser, "The online laboratory: Conducting experiments in a real labor market," Experimental Economics, vol. 14, no. 3, pp. 399-425, 2011.

[35] R. A. Klein, K. A. Ratliff, M. Vianello et al., "Investigating Variation in Replicability," Social Psychology, vol. 45, no. 3, pp. 142-152, 2014.

[36] G. Paolacci, J. Chandler, and P. G. Ipeirotis, "Running experiments on Amazon mechanical Turk," Judgment and Decision making, vol. 5, pp. 411-419, 2010.

[37] P. M. Podsakoff, S. B. MacKenzie, J. Y. Lee, and N. P. Podsakoff, "Common method biases in behavioral research: a critical review of the literature and recommended remedies," Journal of Applied Psychology, vol. 88, no. 5, pp. 879-903, 2003.

[38] M. K. Lindell and D. J. Whitney, "Accounting for common method variance in cross-sectional research designs," Journal of Applied Psychology, vol. 86, no. 1, pp. 114-121, 2001.

[39] S. Jayachandran, S. Sharma, P. Kaufman, and P. Raman, "The role of relational information processes and technology use in customer relationship management," Journal of Marketing, vol. 69, no. 4, pp. 177-192, 2005.

[40] A. F. Hayes, "Partial, conditional, and moderated moderated mediation: quantification, inference, and interpretation," Communication Monographs, vol. 85, no. 1, pp. 4-40, 2018.
[41] A. F. Hayes, A. K. Montoya, and N. J. Rockwood, "The analysis of mechanisms and their contingencies: PROCESS versus structural equation modeling," Australasian Marketing Journal, vol. 25, no. 1, pp. 76-81, 2017.

[42] A. F. Hayes, Introduction to Mediation, Moderation, and Conditional Process Analysis, Guilford, New York, NY, 2013.

[43] C. Hazan and P. R. Shaver, "Romantic love conceptualized as an attachment process," Journal of Personality and Social Psychology, vol. 52, no. 3, pp. 511-524, 1987.

[44] B. C. Feeney and N. L. Collins, "A new look at social support: a theoretical perspective on thriving through relationships," Personality and Social Psychology Review, vol. 19, no. 2, pp. 113-147, 2015.

[45] M. Mikulincer and P. R. Shaver, "Adult attachment orientations and relationship processes," Journal of Family Theory and Review, vol. 4, no. 4, pp. 259-274, 2012.

[46] K. Bartholomew and L. M. Horowitz, "Attachment styles among young adults: a test of a four-category model," Journal of Personality and Social Psychology, vol. 61, no. 2, pp. 226244, 1991.

[47] M. Mikulincer, P. R. Shaver, and D. Pereg, “Attachment theory and affect regulation: the dynamics, development, and cognitive consequences of attachment-related strategies," Motivation and Emotion, vol. 27, no. 2, pp. 77-102, 2003.

[48] J. Crowell, R. C. Fraley, and P. R. Shaver, "Measures of individual differences in adolescent and adult attachment," in Handbook of Attachment: Theory, Research, and Clinical Applications, J. Cassidy and P. R. Shaver, Eds., pp. 599-634, Guilford Press, New York, 2nd Edition edition, 2008.

[49] J. Davila, B. R. Karney, and T. N. Bradbury, "Attachment change processes in the early years of marriage," Journal of Personality and Social Psychology, vol. 76, no. 5, pp. 783-802, 1999.

[50] R. C. Fraley, A. M. Vicary, C. C. Brumbaugh, and G. I. Roisman, "Patterns of stability in adult attachment: an empirical test of two models of continuity and change," Journal of Personality and Social Psychology, vol. 101, no. 5, pp. 974-992, 2011.

[51] T. Umemura, L. Lacinová, K. Kotrčová, and R. Chris Fraley, "Similarities and differences regarding changes in attachment preferences and attachment styles in relation to romantic relationship length: longitudinal and concurrent analyses," Attachment \& Human Development, vol. 20, no. 2, pp. 135159, 2018.

[52] M. F. Lafontaine, A. Brassard, Y. Lussier, P. Valois, P. R. Shaver, and S. M. Johnson, "Selecting the best items for a short-form of the experiences in close relationships questionnaire," European Journal of Psychological Assessment, vol. 32, no. 2, pp. 140-154, 2015.

[53] J. Dion, J. Gervais, N. Bigras, M. E. Blackburn, and N. Godbout, "A longitudinal study of the mediating role of romantic attachment in the relation between child maltreatment and psychological adaptation in emerging adults," Journal of Youth and Adolescence, vol. 48, no. 12, pp. 2391-2402, 2019.

[54] J. Fitzpatrick and M. Lafontaine, "Attachment, trust, and satisfaction in relationships: investigating actor, partner, and mediating effects," Personal Relationships, vol. 24, no. 3, pp. 640-662, 2017.

[55] L. M. Vowels and K. B. Carnelley, "Attachment styles, negotiation of goal conflict, and perceived partner support during 
COVID-19," Personality and Individual Differences, vol. 171, article 110505, 2021.

[56] B. T. McDaniel and M. Drouin, "Daily technology interruptions and emotional and relational well-being," Computers in Human Behavior, vol. 99, pp. 1-8, 2019.

[57] A. Vanden, "Digital wellbeing as a dynamic construct," Communication Theory, vol. 31, no. 4, pp. 932-955, 2020.

[58] S. Khodabakhsh and L. E. Ong, "The impact of partner phubbing on marital quality among married couples in Malaysia: moderating effect of gender and age," Aloma, vol. 39, no. 1, pp. 9-16, 2021.

[59] V. Chotpitayasunondh and K. M. Douglas, "How "phubbing" becomes the norm: the antecedents and consequences of snubbing via smartphone," Computers in Human Behavior, vol. 63, pp. 9-18, 2016.

[60] A. Ivanova, O. Gorbaniuk, A. Blachino et al., "Mobile phone addiction, phubbing, and depression among men and women: a moderated mediation analysis," Psychiatric Quarterly, vol. 91, no. 3, pp. 655-668, 2020.

[61] K. S. Birditt, K. L. Fingerman, and D. M. Almeida, "Age differences in exposure and reactions to interpersonal tensions: a daily diary study," Psychology and Aging, vol. 20, no. 2, pp. 330-340, 2005. 